\title{
Muséologies
}

Les cahiers d'études supérieures

\section{Impressions de voyage : la nouvelle muséologie, du Portugal au Québec}

\section{Jean-François Leclerc}

Volume 2, numéro 1, octobre 2007

URI : https://id.erudit.org/iderudit/1033603ar

DOI : https://doi.org/10.7202/1033603ar

Aller au sommaire du numéro

Éditeur(s)

Association Québécoise de Promotion des Recherches Étudiantes en Muséologie (AQPREM)

ISSN

1718-5181 (imprimé)

1929-7815 (numérique)

Découvrir la revue

Citer ce document

Leclerc, J.-F. (2007). Impressions de voyage : la nouvelle muséologie, du Portugal au Québec. Muséologies, 2(1), 146-155.

https://doi.org/10.7202/1033603ar d'utilisation que vous pouvez consulter en ligne.

https://apropos.erudit.org/fr/usagers/politique-dutilisation/ 


\title{
Impressions de voyage : la nouvelle muséologie, du Portugal au Québec
}

[Par Jean-François Leclerc, directeur du Centre d'histoire de Montréal ]

\begin{abstract}
À la suite d'une formation collégiale en sciences, Monsieur Leclerc obtenait un baccalauréat et une maîtrise en histoire de l'Université de Montréal. Après avoir travaillé comme historien pigiste et obtenu un diplôme en muséologie de l'UQÀM, il s'est consacré à des projets de diffusion liés au patrimoine et à l'histoire. II dirige depuis 1996 le Centre d'histoire de Montréal, une institution muséale de la Ville de Montréal dont la mission est de faire comprendre la ville actuelle en mettant en valeur son histoire et son patrimoine, grâce notamment à certaines actions inspirées de la muséologie sociale. II préside actuellement le Regroupement des musées d'histoire de Montréal et fait partie du comité exécutif de la Société des directeurs de musée de Montréal. jfleclerc@villemontreal.qcica
\end{abstract}


Le voyageur part toujours avec son bagage de références, d'idées préconçues et d'impressions. Le voyage lui permet de les confronter à la réalité, mais, plus encore, lui offre le privilège de prendre une certaine distance avec sa propre société. Le bref moment d'étrangeté vécu au retour est précieux, car il invite à questionner sa propre expérience.

Une telle occasion m'a été donnée en mai dernier, à la faveur d'une invitation à prononcer une conférence d'ouverture sur le thème Quelle gestion pour les musées municipaux?, lors de la $14^{e}$ Rencontre de muséologie municipale tenue du 25 au 27 mai 2006 à Aljezur/Carrapateira (XIV Encontro Museologia Autarquias).
Cette rencontre réunissait, sous l'égide de l'Universidade Lusófona de Humanidades e Tecnologias de Lisbonne ${ }^{[1]}$, les professionnels et les gestionnaires des musées municipaux portugais, de même que des universitaires et des muséologues travaillant dans une perspective de muséologie sociale. Le village d'Aljezur, dans l'Algarve, région touristique bien connue, accueillait les participants au moment où les autorités régionales s'apprêtaient à ouvrir à Carrapateira, non loin de là, un nouveau musée consacré à l'identité des populations locales. Luisa Rogado, muséologue responsable de ce projet pour la municipalité, et Pierre Mayrand, pionnier de la nouvelle muséologie au Québec, avaient organisé la rencontre.
[1]

Le Centro de Estudos de Sociomuseologia (CESMU) fut créé en 1993 pour la promotion de la muséologie et de la recherche, en particulier la muséologie locale et l'écomuséologie. Ce centre dirige un programme de maîtrise en muséologie et publie les Cadernos de Sociomuseologia, principale publication portugaise dédiée à la muséologie. Le CESMU organise régulièrement des rencontres en collaboration avec les universités et les musées brésiliens, ainsi que le MINOM (Mouvement international pour une nouvelle muséologie) et l'ıCOM (Conseil international des musées). 
Pour moi, qui dirige le Centre d'histoire de Montréal (CHM) depuis dix ans, ce fut l'occasion de rencontrer pour la première fois des muséologues qui partageaient une certaine communauté de pensée autour du rôle du musée municipal dans la société. Contrairement au Québec, où les musées gérés et financés directement par les gouvernements municipaux sont l'exception, la majorité des musées portugais sont sous la responsabilité d'administration publique nationale, régionale ou locale. Par son cadre administratif, le Centre d'histoire de Montréal présentait donc pour les muséologues portugais des traits familiers. Par contre, le contexte montréalais pouvait leur paraître un peu plus exotique en raison des modes de gestion éclectiques des institutions muséales par l'administration montréalaise ${ }^{[2]}$ et de la bouillonnante marmite culturelle dans laquelle la métropole est plongée. J'ai tenté de démontrer l'importance pour une ville de pouvoir compter sur ses propres musées, même si leur légitimité est aujourd'hui questionnée. En effet, la muséologie, en particulier la muséologie sociale, peut trouver dans la structure municipale autre chose que des contraintes. Les réseaux internes et externes de la fonction publique, ses expertises diversifiées, sa mission d'assurer aux citoyens un environnement urbain de qualité présentent certes un potentiel important d'action, en autant que les gestionnaires de la Ville et les élus cessent de ne voir dans le musée qu'une institution coûteuse tournée vers le divertissement culturel.

\section{[2]}

Les musées municipaux montréalais sont le Centre d'histoire de Montréal, intégré à l'administration centrale au sein du Service de développement culturel, de la qualité de vie et de la diversité ethnoculturelle (SDCOMVDE), le Musée de Lachine, administré par l'arrondissement de Lachine, le réseau des Museums nature, entité distincte placée sous le SDCOMVDE, et Pointe-àCallière, musée d'archéologie et d'histoire, propriété municipale financée par la Ville mais gérée par un organisme à but non lucratif. 
Je connaissais le Portugal comme destination touristique, mais à peu près pas comme référence muséologique. Notre grand repère européen en ce domaine est évidemment la France, pays des grandes expositions universelles, des musées fondateurs tel le Louvre révolutionnaire ou inspirants comme $\mathrm{La}$ Villette, mais aussi source des textes et des pensées novatrices des Rivière, Desvallée, de Varine et autres auxquels notre langue commune nous donne accès. Le milieu muséologique français nous le rend d'ailleurs assez bien, fasciné par la nord-américanité créative de notre muséologie. Bien qu'ils soient peu revendiqués, les concepts américains font aussi partie de ces références, si nous pensons par exemple à l'influence déterminante de l'interprétation et des centres d'interprétation sur notre muséographie. Le Portugal mériterait d'occuper une place dans le cœur des muséologues québécois qui valorisent le rôle social du travail muséal. En effet, contrairement à ce qu'on peut observer ici, le concept de musée au service des populations suscite encore là-bas des discussions et des débats, même si ses idéaux portés par la "nouvelle muséologie» trentenaire y ont perdu de leur vigueur.
Cette rencontre présentait donc un intérêt certain pour une institution comme le Centre d'histoire de Montréal dont le code génétique comporte une dimension écomuséale. Dès sa création, en 1983, on avait en effet conçu ce centre d'interprétation comme une porte d'entrée destinée à révéler aux Montréalais et aux touristes le sens caché de l'environnement urbain, en faisant apparaître sa profondeur historique. Sa véritable collection était constituée de l'ensemble des patrimoines et des cultures du territoire montréalais. Comme on peut l'imaginer, le concept de porte d'entrée, fonctionnel dans un lieu historique ou naturel clos ou bien délimité, était pour une ville comme Montréal une vue de l'esprit. Faute de ressources suffisantes, il était difficile de faire franchir cette porte virtuelle par une majorité des citoyens auxquels elle était destinée sur un territoire aussi vaste et peuplé. Pour rendre ce concept fonctionnel, il est apparu important que cette "porte" devienne mobile, qu'elle se déplace vers le public, là où il vit. C'est du moins ainsi que nous avons interprété notre mission au début des années 2000 , au moment d'inaugurer notre nouvelle exposition permanente. Le statut municipal du Centre 
d'histoire et la notion de service qui s'y rattache le justifiaient amplement. Il va en effet de soi qu'un service municipal, pour être efficace et universel, doive se déplacer vers les populations ou être établi à proximité, comme c'est le cas notamment des bibliothèques publiques. Notre mobilité a pris la forme d'expositions et d'activités présentées hors du secteur immédiat du centre, le Vieux-Montréal ${ }^{[3]}$. Nous avons privilégié le patrimoine immatériel que constituent la mémoire et l'histoire individuelle des
Montréalais et des Montréalaises, parce que chaque citoyen les préserve, en veillant toujours à les relier à l'histoire collective et aux patrimoines matériel et immobilier. Cette réinterprétation de la dimension écomuséale du CHM s'est poursuivie par la sensibilisation des services municipaux à sa capacité d'agir comme expert en muséologie pour divers projets d'exposition hors les murs ${ }^{[4]}$ et dans le contexte de projets visant à favoriser la résolution de certaines problématiques urbaines ${ }^{[5]}$.

\section{[3]}

Les cliniques de mémoire, les visites Mémoire de ma maison, mémoire de mon quartier et le Musée de la personne Montréal, ainsi que diverses expositions hors les murs ont exploré cette voie avec constance. Pour plus de détails, voir le site du musée : <ville.montreal.qc.ca/chm>.

\section{[4]}

Par exemple, des expositions sur les trente ans des Jeux olympiques à l'hôtel de ville, sur les quarante ans d'Expo 67 à lîle Sainte-Hélène, sur le Dr Norman Bethune au pavillon de Montréal à Shanghai, sans parler de plusieurs expositions itinérantes qui circulent dans les maisons de la culture.
[5]

Une exposition sur les écuries urbaines et le travail de cocher, alors que cette industrie ouristique connaît plusieurs problèmes. 
Ce faisant, je m'inscrivais dans le courant des muséologues qui, depuis plus de trente ans, cherchent d'autres voies que celles portées par la tradition classique des musées et ses versions modernisées. Malgré des valeurs partagées, il demeurait pour moi difficile de m’identifier complètement à la vision du musée (dont le Mouvement international pour une nouvelle muséologie fait toujours la promotion). En effet, comme plusieurs de mes collègues, j’avais connu l'existence de cette "nouvelle muséologie» au cours de ma formation universitaire. Je l'associais spontanément au militantisme social des années 1970 et à l'écomusée, cet héritier le plus visible et connu de la révolution des musées. Les exemples locaux d'écomusée étaient par ailleurs rares - le Fier Monde et la Haute-Beauce - et ceux d'autres pays assez peu connus, sauf peut-être le vénérable Creusot français. Qui avait entendu parler des expériences de Santa Cruz de Rio, de Molinos en Espagne, de Nayarit au Mexique ou de Seixal au Portugal? Les exigences de la nouvelle muséologie, tels la gestion du musée par les populations et son engagement à résoudre des problèmes locaux, me semblaient dépasser la mission de mon institution et ses capacités. Comment en effet une institution municipale liée à une entité politique pouvait-elle assumer la fonction critique inhérente à ce type de muséologie? Au Québec, autour de moi, la "nouveauté" muséale avait plutôt pris la forme d'une muséologie professionnelle, efficace et technocratique, lancée à la conquête du marché des consommateurs culturels. L'appropriation du musée par la population et l'action militante nétaient pas au programme. Les inspirations et les combats de la nouvelle muséologie des années 1970 semblaient s'être fondus dans un syncrétisme muséal non dénué d'une sensibilité envers la communauté, mais qui confondait parfois l'engagement social avec l'opération de marketing, l'enracinement communautaire 
avec les techniques de fidélisation des clientèles et la remise en question de l'objet muséal avec les progrès de la muséographie. Le Québec avait pourtant connu sa propre révolution sociale, comme le Portugal sa révolution politique. Pourquoi, au Portugal, la muséologie sociale avait-elle réussi non seulement à prendre racine, comme ici, mais surtout à trouver une stabilité, des lieux de discussion et d'expérimentation, et à former nombre de muséologues actifs qui revendiquaient cette appartenance ${ }^{[6]}$ ?

Tant le Portugal que le Québec ont vécu au cours des années 1970 une période intense de remises en question à laquelle n'a pas échappé la muséologie. Comme le souligne Pierre Mayrand,

les années 1970, correspondant à la création de l'écomusée et à l'adoption de la Déclaration de Santiago, sont celles de la révolution sociale au Québec et de l'émergence du nationalisme militant (création du Ralliement pour l'indépendance, mesures de guerre, Front de libération du Québec d'allégeance marxiste). Des expériences du mouvement "Dignité" pour la survie des régions menacées, des principes démocratiques liés à la démocratisation culturelle, du projet de société proposé dans la politique de développement culturel et muséal (Musée du Québec en devenir), reposant sur une grille de lecture sociologique, anthropologique et géographique, naissent les premiers écomusées (Haute-Beauce et Fier Monde, rural et urbain jumelés ${ }^{[7]}$.

$\mathrm{Au}$ Québec comme au Portugal, quoique de manière moins affirmée, les partis politique présentaient alors des différences idéologiques
[6]

Pour une population d'environ 10 millions d'habitants, le Portugal compte quelques centaines de musées, en majorité gérés par des administrations publiques. II s'est doté au début des années 2000 d'une loi cadre des musées et d'un réseau (Rede Portuguesa de Museus) qui regroupe environ 120 musées. Ce réseau joue à la fois les fonctions de notre Société des musées québécois pour la formation et l'échange d'expertise, du Conseil des arts et du ministère de la Culture et des Communications du Québec (MCCO) par son pouvoir de reconnaître et de financer les musées selon certains critères professionnels. Pour le reste, le paysage muséal présente quelques similitudes avec le Québec, par la dimension modeste de la plupart des institutions qui sont destinées à mettre en valeur des bâtiments historiques, l'histoire et l'ethnologie locales.
[7]

Pierre MAYRAND.

Cadernos de sociomuseologia, no 22, 2004. <http://cadernosociomuseologia.ulusofona. pt/Arquivo/caderno_22/ cadernos_reflexions_ liminaires.htm>. 
très nettes. À la Révolution tranquille québécoise et au réveil nationaliste et social-démocrate des années 1970, correspond au Portugal la Révolution des œillets d'avril 1974 qui met fin à la dictature du régime Salazar, sonnant le réveil social et communautaire des populations. Par la suite, comme l'écrit Alfredo Tinoco, coordonnateur du regroupement portugais $\mathrm{du}$ Mouvement international pour une nouvelle muséologie, une fois épuisées les formes de participation politique immédiates, les préoccupations de ceux qui sont civiquement actifs se sont tournées vers l'environnement, le social et le culturel. Et ceux-ci ont trouvé dans les muséologies nouvelles un terrain fertile où ils peuvent semer et cueillir les fruits de leur capacité créatrice et de leur volonté de participation dans la gestion collective et critiquement démocratique des problèmes des communautés qui donnent forme et sens au Musée ${ }^{[8]}$.
La nouvelle muséologie est apparue aux partis de gauche comme un outil mobilisateur facilitant le passage d'une société traditionnelle coincée dans des idéologies de droite vers une société plus ouverte. Les partis municipaux portugais étant généralement issus des partis nationaux, ce qui n'est pas le cas au Québec, et partageant les mêmes idéaux et orientations, plusieurs localité ont démontré les mêmes affinités pour cette muséologie et ses projets. Quant aux acteurs les plus dynamiques de la nouvelle muséologie, proches des mouvements socialistes et communistes, ils ont accédé à des postes influents dans l'administration, dans certaines universités et à la direction de musées régionaux.

Il faudrait évidemment faire une analyse comparative moins impressionniste pour établir avec certitude les similitudes et les différences

[8]

MOVIMENTO INTERNACIONAL

PARA UMA NOVA MUSEOLOGIA / MINOM Portugal. "Textes de Muséologie". Cadernos do MINOM, Lisbonne 1992, p. 8. 
entre les parcours historiques des muséologies québécoise et portugaise, en connaissant mieux les forces et les faiblesses de chacune. Le voyageur perplexe que je fus au retour du Portugal s'est tout de même demandé où se trouve aujourd'hui la nouvelle muséologie québécoise dans sa forme sociale. Si elle ne se manifeste plus depuis des lustres par la création d'écomusées, elle anime l'action de nombreux muséologues qui travaillent au sein de musées «intermédiaires", pour reprendre le terme de Pierre Mayrand, ces institutions mixtes issues de la vieille tradition muséale ou de création plus récente. Plusieurs d'entre eux, sans renier les outils d'entrepreneurs culturels acquis au cours de la professionnalisation des musées, cherchent un engagement personnel et institutionnel plus profond. La muséologie sociale québécoise est certes moins revendicatrice et contestataire que ne le rêvaient probablement ses acteurs de la première heure. Les causes qu'elle défend sont très souvent héritées du libéralisme de droit, du nationalisme civique et de la social-démocratie, véhiculés par la Révolution tranquille québécoise et ses suites, sans oublier l'écologie et le développement durable. Linclusion et le respect des minorités, l'interculturalité, la défense des droits collectifs de la nation québécoise, le respect de la liberté individuelle, l'égalité des chances, la protection du patrimoine et de l'environnement paraissent garants d'une certaine paix sociale et sont même reconnus par la plupart des pouvoirs publics. Ce consensus n'est pas très propice à la mobilisation ni aux éclats, mais il permet aux projets de muséologie sociale de trouver leur place, même dans des institutions plus conservatrices. Cette dissémination sans heurts ne suffit pourtant pas pour qu'elle évolue. L'une des faiblesses de cette muséologie est, à mon avis, de ne pouvoir se voir et prendre conscience de son impact comme de ses faiblesses, faute de forum où échanger les enseignements tirés de nombreuses expériences de terrain. Les «laboratoires de la transformation sociale ${ }^{\left[{ }^{[9]}\right.}$ " que sont parfois nos musées gardent trop souvent leurs portes fermées. Le Mouvement international pour une nouvelle muséologie rattaché à l'ICOM pourrait jouer le rôle de rassembleur et de lieu d'échange. Mais, faute de moyens et d'organisation stable, hors du Portugal où il semble mieux structuré et appuyé et hors du cercle des fidèles et des 
sympathisants de la première heure, il a du mal à faire connaître ses valeurs pourtant partagées sans le savoir par de nombreux muséologues de terrain. Si j'en juge par ma propre perception, l'image du MINOM paraît encore trop étroitement associée à certaines formes muséales ou à certains parti pris, même si, en réalité, comme le soulignait Alfredo Tinoco, le MINOM «ne reconnaît ni une méthodologie, ni une pratique muséologique 'nouvelle' qui puisse servir de repère universel " ${ }^{[10]}$. Au-delà des clichés, il y a donc une place dans ce mouvement pour ceux qui cherchent des voies nouvelles d'engagement qui répondent autrement aux questions : pourquoi un musée, à quelle fin et pour qui ? Encore faut-il la prendre.
La muséologie sociale québécoise cherche à s'inscrire dans l'actualité, est sensible aux préoccupations des populations, soucieuse non seulement de conserver, d'instruire et de divertir, mais d'avoir un impact sur la vie et les valeurs des communautés. Elle essaie petit à petit, par essais et erreurs, de changer les manières de gérer les musées, de voir et de présenter les collections, d'être en relation avec le public, avec les citoyens, avec la communauté. Qui sait si ses expériences ne parviendront pas à réaliser, sous une forme plus discrète mais bien réelle, les rêves encore inspirants des fondateurs de la nouvelle muséologie?

Montréal, 16 août 2007

[10]

De la même façon, on ne reconnaît ni une méthodologie, ni une pratique muséologique 'nouvelle' qui puisse servir de repère universel ; par contre, nous nous reconnaissons dans des expériences aussi diverses que l'écomuséologie, la muséologie populaire, le musée local, très souvent d'initiative municipale, ou la muséologie communautaire, entre autres, sans jamais avoir établi une rupture nette avec des formes de réanimation ou de socialisation qui se développent parfois même dans les musées les plus traditionnels. Tinoco ("Textes de muséologie", p. 8) définit le musée comme pouvant être un «instrument d'apprentissage culturel et social et un laboratoire de transformation sociale" Mayrand (op. cit) parle quant à lui de "l'écomusée dans sa version la plus actuelle d'écomusée communautaire ou d'écomusée communautaire territorial, comme une organisation à vocation socioculturelle, utilisant l'histoire et l'exposition, l'éducation populaire, comme les outils privilégiés d'un projet de connaissance de soi, de développement harmonisé et d'ouverture sur le monde" (<http://cadernosociomuseologiaulusofonapt/Arquivo/ caderno_22/cadernos_ reflexions_liminaires.htm $>$ ) 